\title{
Single-particle-picture breakdown in laterally weakly confining GaAs quantum dots
}

\author{
Daniel Huber $\odot,{ }^{1,2, *}$ Barbara Ursula Lehner, ${ }^{1,2}$ Diana Csontosová $\odot,{ }^{3,4}$ Marcus Reindl $\odot,{ }^{1}$ Simon Schuler, ${ }^{1}$ \\ Saimon Filipe Covre da Silva $\odot,{ }^{1}$ Petr Klenovský $\odot,{ }^{3,4, \dagger}$ and Armando Rastelli $\odot^{1, \$}$ \\ ${ }^{1}$ Institute of Semiconductor and Solid State Physics, Johannes Kepler University Linz, Altenbergerstrasse 69, 4040 Linz, Austria \\ ${ }^{2}$ Secure and Correct Systems Lab, Linz, Institute of Technology, Altenbergerstrasse 69, 4040 Linz, Austria \\ ${ }^{3}$ Department of Condensed Matter Physics, Faculty of Science, Masaryk University, Kotlářská 267/2, 61137 Brno, Czech Republic \\ ${ }^{4}$ Czech Metrology Institute, Okružní 31, 63800 Brno, Czech Republic
}

(Received 11 September 2019; revised manuscript received 20 November 2019; published 13 December 2019)

\begin{abstract}
We present a detailed investigation of different excitonic states weakly confined in single GaAs/AlGaAs quantum dots obtained by the Al droplet-etching method. For our analysis we make use of temperature-, polarization-, and magnetic-field-dependent $\mu$-photoluminescence measurements, which allow us to identify different excited states of the quantum dot system. Besides that, we present a comprehensive analysis of $g$-factors and diamagnetic coefficients of charged and neutral excitonic states in Voigt and Faraday configuration. Supported by theoretical calculations by the configuration-interaction method, we show that the widely used single-particle Zeeman Hamiltonian cannot be used to extract reliable values of the $g$-factors of the constituent particles from excitonic transition measurements.
\end{abstract}

DOI: 10.1103/PhysRevB.100.235425

\section{INTRODUCTION}

Within the past few years, GaAs quantum dots (QDs), obtained with the droplet-etching method [1-3], emerged as a promising source of nonclassical states of light, such as single photons with a strongly suppressed multiphoton emission probability [4], highly indistinguishable photon states [5-8], and single polarization entangled photon-pairs with a near unity degree of entanglement [5,9-11].

Only recently was it realized that excitons must be weakly confined in these QDs, as the measured ground-state exciton $(X)$ lifetimes of about 250 ps $[5,9,10]$ are significantly lower than the minimum lifetime expected for a strongly confining system (440 ps) [6,12]. The morphology of such GaAs/AlGaAs QDs features in fact a lateral extension, which is much larger than the free-exciton Bohr radius in GaAs $[3,5,6]$. Therefore, the excitonic states are laterally weakly confined and the Coulomb interaction between the charge carriers is supposed to overwhelm quantum confinement effects. In turn, the weak confinement regime is partly responsible for the excellent optical properties of GaAs QDs, as the short lifetime and large in-plane extension of the wave function limit the influence of dephasing and structural anisotropies within the QD [13].

We note that a standard model system for the weak confinement regime is represented by GaAs QDs formed at thickness

\footnotetext{
*daniel.huber@jku.at

†klenovsky@physics.muni.cz

‡armando.rastelli@jku.at
}

Published by the American Physical Society under the terms of the Creative Commons Attribution 4.0 International license. Further distribution of this work must maintain attribution to the author(s) and the published article's title, journal citation, and DOI. fluctuations in thin GaAs/AlGaAs quantum wells [14], which, however, suffer from a poor control of the lateral confinement. Furthermore, the energy separation between confined states and delocalized states is small. In contrast to that, dropletetched GaAs QDs avoid these issues and provide an ideal system to study the properties of weakly confined excitons.

In addition to possible applications of QDs as singlephoton and entangled photon-pair sources, the spin states of charges confined in a QD may serve as qubits for quantum technology $[15,16]$. Moreover, semiconductor QDs could provide a link between photonic and spin qubits via photon-tospin conversion [17]. Such complex applications require a detailed knowledge about the response of an excited state in the QD to an external magnetic field, which is described by its $g$-factor and the diamagnetic coefficient. Furthermore, an individual engineering of the contribution of electrons $\left(e^{-}\right)$ and holes $\left(h^{+}\right)$within a complex to the overall $g$-factor is desired [18-20]. Hence, for engineering the magnetic properties of a $\mathrm{QD}$, precise measurements are crucial. Usually, to extract the $g$-factors of $e^{-}$and $h^{+}$confined in single QDs from photoluminescence (PL) measurements, a single-particle (SP) Zeeman Hamiltonian [21] is employed [18,19,22,23] (for details about the method, we refer the interested reader to Ref. [24]). However, it is questionable whether one can apply such an approach to weakly confining QDs. The knowledge of the magneto-optical properties of GaAs/AlGaAs QDs obtained by droplet etching is restricted to only a few works [25-27], and a comprehensive analysis is to the best of our knowledge missing.

In this work, we first study various excitonic transitions in GaAs QDs via polarization-resolved and temperaturedependent $\mu$-PL measurements, which allow us to identify several charged states such as the positive $\left(X^{+}\right)$and negative $\left(X^{-}\right)$trion as well as some of their excited states. We then present a comprehensive study on the 


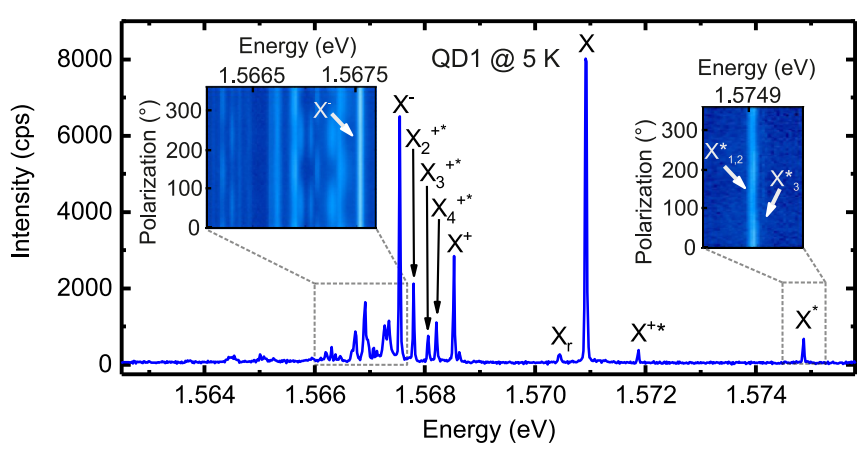

FIG. 1. Spectrum of a representative quantum dot (QD1) under above-band-gap excitation at $5 \mathrm{~K}$. The identified recombination channels are labeled within the figure; see the text for details. The insets show color-coded $\mu$-PL spectra of transitions within the gray dotted boxes.

magneto-optical properties of the GaAs/AlGaAs QD system. We apply magnetic fields along the QD growth direction [001] (Faraday configuration) and along the [110] direction (Voigt configuration), and we extract the diamagnetic coefficients and $g$-factors of several excited states in the GaAs QDs. To gain more insight into the physical properties of our QDs under an external magnetic field, we complement our experimental study with calculations using the configurationinteraction (CI) method [28-31]. The theoretical results are in good agreement with the experimental data, and they confirm that correlation effects among the confined carriers have a significant influence on the magnetic properties. Finally, we demonstrate by experiment and CI calculations that a SP picture, which turns out to be adequate in the case of strongly confining QDs, leads to poor results in a weakly confining system [21,24,32].

\section{EXPERIMENTAL RESULTS}

\section{A. Polarization-resolved and temperature-dependent $\mu$-PL measurements}

We begin by characterizing the optical transitions of our GaAs/AlGaAs QDs. The details on the sample growth are given in Ref. [10]. For the excitation, we use a $532 \mathrm{~nm}$ continuous-wave laser, which is focused on the sample through an aspheric lens with a 0.65 numerical aperture. The low QD density $\left(\sim 2 \times 10^{7} \mathrm{~cm}^{-2}\right)$ allows us to address the emission of single QDs. In above band-gap excitation, $e^{-}$and $h^{+}$are mainly generated in the barrier material of the QD, where the carriers subsequently diffuse, partly get trapped by the QD confinement potential, and relax to the low-energy levels. The spectrum of a representative QD is shown in Fig. 1. The spectral position of $X$ within the cluster of lines is well known from previous experiments $[3,5,6,10]$. It has two orthogonally polarized components, which are typically nondegenerate in energy due to the fine-structure splitting [21]. The biexciton state is not visible under the used excitation conditions, which we attribute to competition with other configurations due to a slow relaxation of carriers to the ground state via multiple acoustic phonons $[5,6]$.

Above-band-gap excitation provides an interesting feature to identify some of the observed transitions: The mobility of

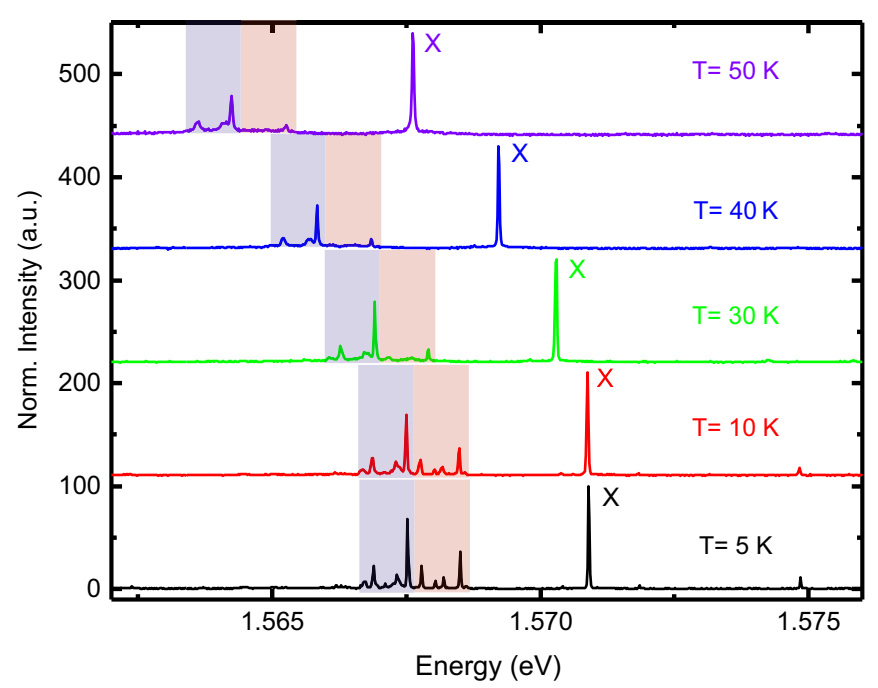

FIG. 2. Normalized temperature-dependent $\mu$-PL spectra of a representative quantum dot (QD1). The quantum dot is excited by a $532 \mathrm{~nm}$ continuous-wave diode laser above the AlGaAs barrier band gap.

$e^{-}$and $h^{+}$within the AlGaAs barrier influences the formation probability of the different charge complexes. The mobility is reduced due to scattering events mainly with ionized impurities and phonons, such that the larger scattering cross section of the $h^{+}$compared to the $e^{-}$plays a significant role in the spectral response [33]. We demonstrate this claim by performing a temperature-dependent $\mu$-PL measurement. By increasing the temperature, the mobility of the $e^{-}$increases compared to that for the $h^{+}$, and we expect that $h^{+}$-dominated complexes (e.g., $X^{+}$) are formed less often than $e^{-}$-dominated ones (e.g., $\left.X^{-}\right)$.

Figure 2 shows temperature-dependent $\mu$-PL spectra for temperatures between 5 and $50 \mathrm{~K}$. The spectra of a representative QD (QD1) are normalized with respect to the intensity of the $X$ transition. On the low-energy side of the $X$, transitions with energies $>1.5675 \mathrm{eV}$ (see the red area in Fig. 2) drop fast in intensity with increasing temperature until they completely disappear. In contrast, transitions with energy $<1.5675 \mathrm{eV}$ (see the blue area in Fig. 2) are less sensitive to a temperature change.

By combining the temperature dependence with polarization-resolved $\mu$-PL measurements, we can now discuss the origin of several transitions in Fig. 1. While the SP picture is poorly suitable for describing the excitonic states of weakly confining QDs [6], we stick to that for the moment to provide an intuitive description of the charge configuration of the individual states. On the low-energy side of $X(<1.570918 \mathrm{eV}$ for QD1 at $5 \mathrm{~K})$ we first find a transition labeled as $X_{r}$. This feature consists of two linearly polarized lines with an energy splitting and polarization orientation equal to those of $X$, and it disappears under quasiresonant excitation. We thus attribute it to a quantized energy jitter of the $X$ stemming from charging and uncharging of a defect in the vicinity of the QD. At an energy of $1.568524 \mathrm{eV}$ we then find the $X^{+}$transition and at 1.567542 we find the $X^{-}$transition. We can distinguish between those by the different temperature trend seen in Fig. 2. The trions 
have no measurable polarization splitting as expected for a Kramers doublet [34]. Between the trions we observe three additional transitions, which are, according to the temperature-dependent measurements, $h^{+}$-dominated. We thus attribute these lines to the excited states of $X^{+}$(hot trions) [33-36]. In the SP picture, the simplest hot trion is described by an $e^{-}-h^{+}$pair in the $s$-shell and an extra $h^{+}\left(e^{-}\right)$ in the $p$-shell. We want to point out that in a more realistic picture including carrier interactions, the situation is not that trivial (see Sec. VI of the Supplemental Material [37]). In most of the QD systems, it is difficult to observe radiative transitions from an excited trion as that is converted rapidly to a ground-state trion via nonradiative relaxation. However, the slow relaxation rates between the energy levels in our QDs allow us to study these transitions in more detail [6]. Due to exchange interaction, the excited positive trion splits into four degenerated doublets [34,35]:

$$
\begin{gathered}
\left|X_{4}^{+*}\right\rangle= \begin{cases}\uparrow_{s}\left(\uparrow_{s} \Downarrow_{p}-\Downarrow_{s} \Uparrow_{p}\right), & J_{z}=+1 / 2, \\
\downarrow_{s}\left(\Uparrow_{s} \Downarrow_{p}-\Downarrow_{s} \Uparrow_{p}\right), & J_{z}=-1 / 2,\end{cases} \\
\left|X_{3}^{+*}\right\rangle= \begin{cases}\uparrow_{s}\left(\Uparrow_{s} \Downarrow_{p}+\Downarrow_{s} \Uparrow_{p}\right), & J_{z}=+1 / 2, \\
\downarrow_{s}\left(\uparrow_{s} \Downarrow_{p}+\Downarrow_{s} \Uparrow_{p}\right), & J_{z}=-1 / 2,\end{cases} \\
\left|X_{2}^{+*}\right\rangle= \begin{cases}\downarrow_{s} \Uparrow_{s} \uparrow_{p}, & J_{z}=(+5 / 2), \\
\uparrow_{s} \Downarrow_{s} \Downarrow_{p}, & J_{z}=(-5 / 2),\end{cases} \\
\left|X_{1}^{+*}\right\rangle= \begin{cases}\uparrow_{s} \uparrow_{s} \Uparrow_{p}, & J_{z}=(+7 / 2), \\
\downarrow_{s} \Downarrow_{s} \Downarrow_{p}, & J_{z}=(-7 / 2),\end{cases}
\end{gathered}
$$

where $\uparrow_{i}, \downarrow_{i}\left(\Uparrow_{i}, \Downarrow_{i}\right)$ describe the $e^{-}\left(h^{+}\right)$spin configuration in the shell $i \in\{s, p\}$, and the number in the parentheses gives the total angular momentum projection on the quantization axis under the simplified assumption that holes in the $s$ - and $p$-states have a pure heavy-hole $(\mathrm{HH})$ character (with $J_{z}=$ $\pm 3 / 2$ ). The singlet state $\left(X_{4}^{+*}\right)$ and two of the triplet states $\left(X_{3}^{+*}\right.$ and $\left.X_{2}^{+*}\right)$ emit a single photon when the $s$-shell $e^{-}-h^{+}$ pair recombines, while the remaining triplet state $\left(X_{1}^{+*}\right)$ is forbidden due to dipole selection rules. Since the energetic ordering of $X_{2}^{+*}-X_{4}^{+*}$ is nontrivial, we follow the labeling given in Refs. [35,36].

Below the $X^{-}$we observe several transitions, which, according to the temperature trend, are $e^{-}$-dominated. Nevertheless, a detailed analysis of these states is not possible in our measurements due to the limited spectral resolution $(\approx 25 \mu \mathrm{eV})$. A polarization-resolved $\mu$-PL measurement (see the inset of Fig. 1) shows nonpolarized lines as well as doublets of orthogonally polarized lines. We speculate that these states belong to the excited $X^{-}$and/or to multiple negatively charged states.

At energies above the $X$ transition we observe two transitions $\left(X^{+*}\right.$ and $\left.X^{*}\right)$, which disappear with increasing temperature (see Fig. 1). The $X^{+*}$ at $1.571869 \mathrm{eV}$ does not show any polarization splitting. We conclude that this is a transition of an excited $X^{+}$where (again in a simplified SP picture) the $h^{+}$in the $p$-shell recombines with an $e^{-}$in the $s$-shell. The $X^{*}$ is a complex of three transitions (see the inset of Fig. 1), where two $\left(X_{1,2}^{*}\right.$ at $\left.1.574865 \mathrm{eV}\right)$ have similar intensities and are orthogonally polarized with an energy splitting of $5 \mu \mathrm{eV}$. The third transition $\left(X_{3}^{*}\right.$ at $\left.1.574931 \mathrm{eV}\right)$ is linearly polarized and lower in intensity by a factor $\sim 15$. This is an excited complex of the bright $X$ doublet (total angular momentum of \pm 1 ) and one component of the dark $X$ doublet (total angular momentum of \pm 2 ), whereby the $h^{+}$of the complex is situated in the $p$-shell. According to our calculations (discussed later on), we attribute the brightening of one of the nominally dark states to the fact that the $p$-state holes have 20\% light-hole (LH) character, which allows for a weak dipole transition (see Sec I of the Supplemental Material [37]) [25]. For clarity, we label the whole transition complex with $X^{*}$ instead of labeling each transition individually.

\section{B. Magneto-optical properties of GaAs QDs}

We continue our study by investigating the magnetic response of the excitonic states discussed above. The QD sample is mounted in a He bath cryostat equipped with a superconducting vector magnet. In a Faraday configuration, the magnetic-field vector is aligned along the [001] crystal axis (growth direction), which we label as $z$. Due to the inplane symmetry of the QDs, we restrict our measurements in a Voigt configuration to magnetic fields along the [110] crystal axis only, which we label as $x$. The magnetic field alters the emission properties of the QD, where (i) the diamagnetic shift and (ii) the Zeeman effect are the dominant processes.

In (i) the magnetic field induces a magnetic moment and changes the energy of a state in first approximation according to

$$
\Delta E=\gamma B^{2},
$$

where $\gamma$ is the diamagnetic coefficient. For the neutral exciton, the diamagnetic coefficient probes the spatial extent of the excitonic wave function, which depends on the spatial confinement and interactions between the confined particles [38-40]. Hence, it is obvious that the diamagnetic shift is a directiondependent quantity. The parabolic behavior from Eq. (5) is only valid in the weak-field limit, where the magnetic length $l_{M}=\sqrt{\frac{\hbar}{e B}}$ is larger than the spatial extent of the wave function $l_{\mathrm{wf}}$ [41]. Note that the magnetic length is $l_{M} \approx 15 \mathrm{~nm}$ at a magnetic field of $3 \mathrm{~T}$, while the excitonic wave function may exceed this value in our GaAs QDs (the QDs have a base diameter of $\approx 60 \mathrm{~nm}$ and a height of $\approx 10 \mathrm{~nm}$ ), such that the diamagnetic shift can deviate from the $B^{2}$ dependence.

In (ii) a magnetic field along $z$ lifts the spin degeneracy, while a field along $x$ also breaks the symmetry of the system introducing a coupling between different states (for example between dark and bright $X$ ) [21,24]. The relation between magnetic field and splitting (mixing) is characterized by the $g$-tensor, whereby we probe its elements along $x\left(g_{x}\right)$ and $z$ $\left(g_{z}\right)$ [24].

The Zeeman effect in QDs is commonly described by a SP Zeeman Hamiltonian, where the $g$-factor is a combination of $e^{-}$and $h^{+} g$-factors [21,24]. However, we find in the following that in the weak confinement regime this approximation is not valid.

Figure 3 shows the shift of the transition lines of QD1 versus the magnetic field between 0 and $2.5 \mathrm{~T}$ applied in the $x$ - and $z$-direction, respectively. To get full insight into the magnetic properties of the different transitions, we additionally record polarization-resolved spectra versus magnetic- 


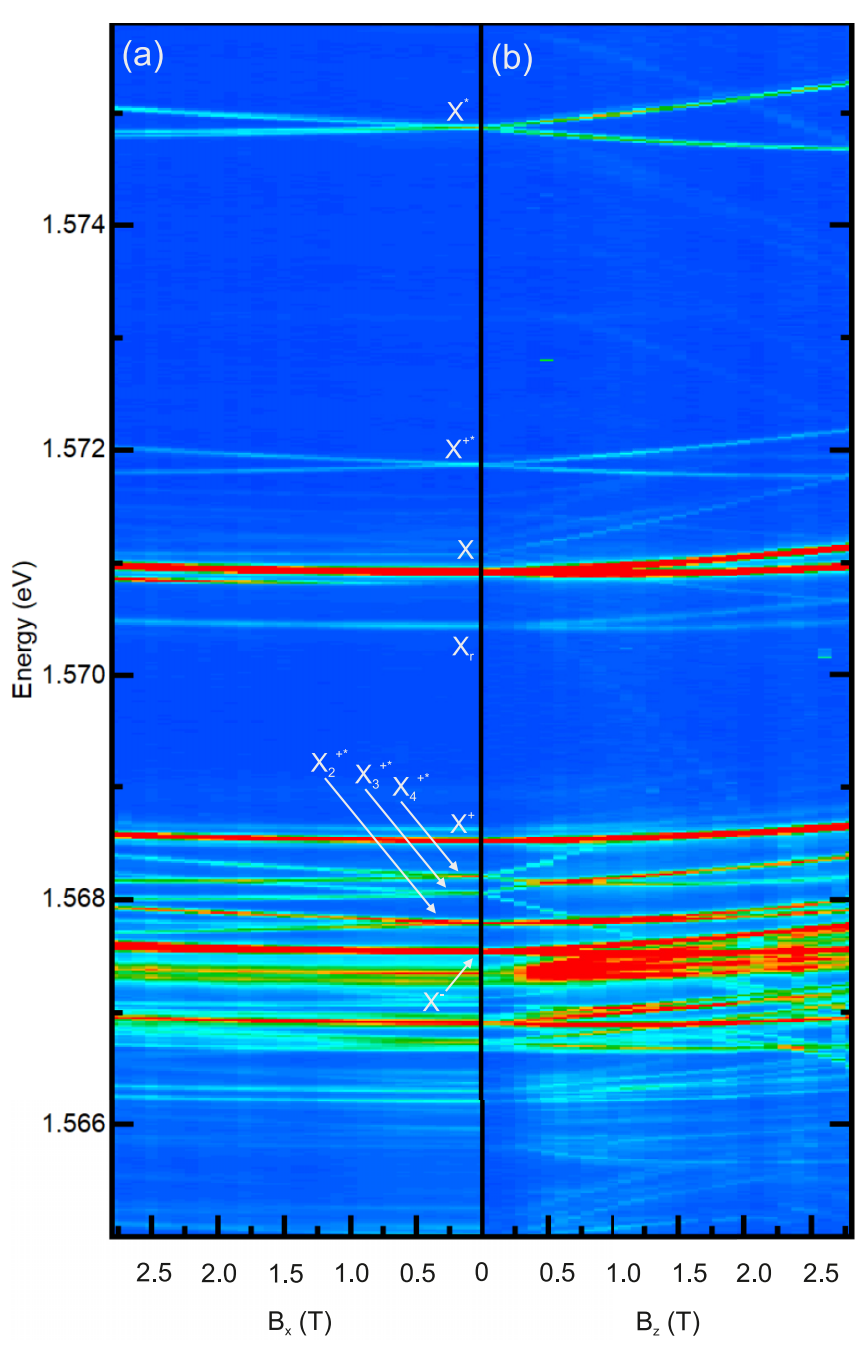

FIG. 3. $\mu$-PL spectra under a magnetic field of a representative QD (QD1) applied along (a) [110] and (b) [001] crystal direction.

field strength $(B)$. Finally, we use these data to extract the diamagnetic coefficients and $g$-factors by fitting the energy shifts with the following model [42]:

$$
E_{\uparrow / \downarrow}=E_{0}+\gamma B^{2} \pm \frac{1}{2} \sqrt{S^{2}+\left(g_{0}+g_{2} B^{2}\right)^{2} \mu_{B}^{2} B^{2}},
$$

where $\uparrow / \downarrow$ label the two Zeeman-split states, $E_{0}$ is the energy of the transition at $B=0, g_{0}$ is the $g$-factor, $g_{2}$ is a secondorder term, $S$ is a possible initial fine-structure splitting, and $\mu_{B}$ is the Bohr magneton. For convenience, we separate the diamagnetic shift and the Zeeman interaction during fitting by using $\frac{1}{2}\left(E_{\uparrow}+E_{\downarrow}\right)$ to obtain $\gamma$ and $E_{\uparrow}-E_{\downarrow}$ to obtain $g_{0}$ and $g_{2}$, respectively. In a Faraday configuration, the model in Eq. (6) can be used to fit satisfactorily all transitions. However, in Voigt geometry the situation is more complicated due to mixing between the states. We can still use Eq. (6) to fit the $X$ transition for small fields, where the bright-dark mixing is negligible, and also for $X_{2}^{+*}-X_{4}^{+*}$, each splitting into two circularly polarized components. The $X^{+}$and $X^{-}$, which are not much influenced by the exchange interaction, split instead into four linearly polarized components each, and thus cannot be described by Eq. (6). Therefore, we follow the approach outlined in Ref. [19] and calculate the $g$-factor according to

$$
g_{x}=\frac{E_{1}-E_{2}}{\mu_{B} B},
$$

where $E_{1}\left(E_{2}\right)$ is the highest- (lowest-) energy component of the trion quadruplet. Note that we do not separate the $g$-factor in the $e^{-}$and $h^{+}$contribution as shown in Ref. [19]. Hence, Eq. (7) has to be considered as a phenomenological model describing well the trend of the obtained experimental and theoretical data. We use the same model to extract the $g$-factors of $X^{+*}$ and $X^{*}$, which are found to split into four linearly polarized components. In Table I, we summarize the results for a representative QD (QD1).

We observe pronounced anisotropies of the $g$-factors along the $x$ - and $z$-direction. This phenomenon has been observed also for other QD systems [43] and can be qualitatively explained in the SP picture following the arguments of Ref. [44]: the conduction band $e^{-} g$-factor is approximately isotropic due to the underlying $s$-type atomic orbitals; this is not true for the valence band $h^{+}[18,24,45]$ as the angular momentum of the $\mathrm{HH}$ Bloch state has only a projection along the $z$-direction. Therefore, the $h^{+} g$-factor is $g_{h} \approx 0$ along $x$, while along $z, g_{h} \gg 0$ is expected [46]. In the SP picture, the $g$-factor of a state is considered as a combination of the individual $e^{-}$ and $h^{+} g$-factors forming the state. The $h^{+}$ground state in our QDs has a dominant HH character [25], so that, depending on the signs of the single-particle $g$-factors, the situation $g_{z}>g_{x}$ arises for $X, X^{+}$, and $X^{-}$. The charged states $X^{+}$ and $X^{-}$already allow us to observe a discrepancy from the SP picture. Within that simple model, the $g$-factor of the $X^{+}$ and $X^{-}$is given by $g_{X^{+}}=g_{e, 1}+g_{h, 2}$ and $g_{X^{-}}=g_{e, 2}+g_{h, 1}$, respectively, whereby $g_{e(h), 1}$ is the single $e^{-}\left(h^{+}\right) g$-factor in the initial state and $g_{e(h), 2}$ is the $g$-factor of the remaining $e^{-}\left(h^{+}\right)$[47]. Since the involved $e^{-}$and $h^{+}$occupy exclusively the ground $s$ levels, we would expect a similar $g$-factor for $X^{+}$ and $X^{-}$, which, however, is not confirmed by our experimental data. Furthermore, $X^{+}$does not even show a significant $x-z$ anisotropy. We want to point out that $X^{+}, X^{-}$, and $X$ are linearly polarized under $z$-field, which is not expected from SP theory (see Sec. II of the Supplemental Material [37]) [21].

The transitions $X^{*}, X^{+*}$, and $X_{2}^{+*}-X_{4}^{+*}$ show a larger $g_{x}$. Interestingly, the $g$-factor anisotropy is reversed for $X_{2}^{+*}$, i.e., $g_{x}>g_{z}$. As discussed above, in a SP picture, the $X_{2}^{+*}-X_{4}^{+*}$ transitions stem from the recombination of a ground-state electron with a ground-state hole in the presence of a hole in the first excited state. The pronounced difference in the $g$-factor anisotropy compared to $X^{+}$clearly indicates that the extra $h^{+}$is by far not a simple "spectator" and that its presence and properties (in particular its significant LH content) have profound effects on the response of the resulting exciton to magnetic fields. We find that $X^{*}, X^{+*}, X_{4}^{+*}$, and $X_{3}^{+*}$ have larger values of $g_{z}$ compared to the ground-state transitions $X$, $X^{+}$, and $X^{-}$. We attribute this to the LH-HH coupling [48]. In particular, the large $g_{z}$ value of $X_{3}^{+*}$ leads to a crossing of one of its components with the $X^{+}$states at moderate magnetic fields. For fields above the crossing point such a component disappears, possibly because of efficient relaxation to the lower-energy $X^{+}$state. Furthermore, we observe a coupling between the recombination channels of $X^{*}$ as expected for a 
TABLE I. The table summarizes fitted diamagnetic coefficients and $g$-factors for the excitonic transitions in a representative quantum dot (QD1) and the CI calculation results for a magnetic field applied along the [110] $(x)$ and [001] (z) directions.

\begin{tabular}{|c|c|c|c|c|c|c|c|c|c|c|c|c|c|}
\hline QD1 & $\gamma_{x}\left(\frac{\mu \mathrm{eV}}{T^{2}}\right)$ & $g_{x}$ & $g_{2, x}\left(T^{-2}\right)$ & $\gamma_{z}\left(\frac{\mu \mathrm{eV}}{T^{2}}\right)$ & $g_{z}$ & $g_{2, z}\left(T^{-2}\right)$ & CI & $\gamma_{x}\left(\frac{\mu \mathrm{eV}}{T^{2}}\right)$ & $g_{x}$ & $g_{2, x}\left(T^{-2}\right)$ & $\gamma_{z}\left(\frac{\mu \mathrm{eV}}{T^{2}}\right)$ & $g_{z}$ & $g_{2, z}\left(T^{-2}\right)$ \\
\hline$X^{*}$ & $5.9(5)$ & $1.61(3)$ & & $12.7(3)$ & 3.74 (1) & -0.01 & $X^{*}$ & 4.56 & 1.7 & 0.2 & 14.7 & 2.57 & 0.74 \\
\hline$X^{+*}$ & $6.4(5)$ & $1.45(3)$ & & $11.5(9)$ & $2.58(1)$ & $-0.01(1)$ & $X^{+*}$ & 6.92 & 0.46 & 0.03 & 8.03 & 0.58 & 0.12 \\
\hline$X^{+}$ & $7.6(7)$ & $0.18(5)$ & & $16.29(4)$ & $0.13(1)$ & 0.001 (1) & $X^{+}$ & 5.8 & 0.48 & 0.01 & 16.17 & 0.59 & 0.12 \\
\hline$X_{4}^{+*}$ & $10.4(3)$ & $1.41(1)$ & $0.003(1)$ & 139 (1) & $6.33(1)$ & 0.18 (1) & $X_{4}^{+*}$ & 4.17 & 1.69 & 0.04 & 96.11 & 2.93 & 0.87 \\
\hline$X_{3}^{+*}$ & $10.6(9)$ & $1.13(5)$ & $0.18(4)$ & $-94(1)$ & $8.27(1)$ & $-0.33(1)$ & $X_{3}^{+*}$ & 2.07 & 1.7 & 0.07 & -55.32 & 3.31 & 1.03 \\
\hline$X^{-}$ & $6(1)$ & $0.33(3)$ & & $16.9(5)$ & $1.42(1)$ & $-0.046(1)$ & $X^{-}$ & 4.46 & 0.919 & 0.0009 & 15.61 & 2.12 & 0.06 \\
\hline
\end{tabular}

complex including a dark state (see Sec. I of the Supplemental Material [37]) [49].

In contrast to our expectations, the diamagnetic shifts are well fitted by Eq. (5) also for high magnetic fields. We find $\gamma_{x}<\gamma_{z}$ (except for $X_{3}^{+*}$ ). For the neutral $X$ this is qualitatively expected, as the wave function is strongly (weakly) confined in the $z(x)$ directions, leading to a small (large) $\gamma_{x}\left(\gamma_{z}\right)$. For a charged state, a carrier remains in the QD after $e^{-}-h^{+}$ recombination. Hence, the measured $\gamma$ depends on the localization of the initial as well as the final state. We observe a $\gamma_{x}$, which is significantly larger for $X_{4}^{+*}$ and $X_{3}^{+*}$ compared to the other states and an unexpected high $\gamma_{z}$ for $X_{4}^{+*}$. Furthermore, we find a negative diamagnetic coefficient for $X_{3}^{+*}$. This is known as an anomalous diamagnetic shift and was observed for negative trions in InAs/GaAs QDs with weakly confined $e^{-}$in the conduction band (see Sec. IV of the Supplemental Material [37]) [41]. In our QDs, we ascribe the anomalous diamagnetic shift to an initial state, which is more localized along $x$ than the remaining hole after recombination.

To demonstrate that the obtained results are not a feature of a single QD, we extended our study to several QDs (see Sec. III of the Supplemental Material [37]). It turns out that most of the magneto-optical properties are similar, however we observe significant differences for the excited trion states $X_{2}^{+*}-X_{4}^{+*}$, indicating a strong dependence on the structural properties of the QD.

As discussed above, the magnetic properties of the trions contradict the SP model. We now demonstrate that the SP Zeeman Hamiltonian is not even sufficient to describe the magnetic response of a ground state $X$. We follow Ref. [24], where the Hamiltonian of a neutral exciton under an in-plane magnetic field in the total angular momentum basis is given by

$$
H_{B}^{x}=\frac{1}{2}\left[\begin{array}{cccc}
\delta_{0} & \delta_{1} & \epsilon_{e} & \epsilon_{h} \\
\delta_{1} & \delta_{0} & \epsilon_{h} & \epsilon_{e} \\
\epsilon_{e} & \epsilon_{h} & -\delta_{0} & \delta_{2} \\
\epsilon_{h} & \epsilon_{e} & \delta_{2} & -\delta_{0}
\end{array}\right]
$$

where $\delta_{0}$ is the splitting between bright and dark exciton states, $\delta_{1}$ is the exchange splitting between the bright states (fine-structure splitting), $\delta_{2}$ is the splitting between the dark states, and $\epsilon_{e(h)}=\mu_{B} B_{x} g_{e(h), x}$, with $g_{e(h), x}$ the $e^{-}\left(h^{+}\right) g$ factor in the $x$-direction. Due to field-induced bright-dark $X$ coupling, in total four dipole transitions are possible. We label the transitions as $X_{b 1,2}$ and $X_{d 1,2}$, where $X_{b i}\left(X_{d i}\right)$ are the transitions of the $X$ complex, which are bright (dark) at $B=0$, and $i=1,2$ are the respective orthogonally polarized components. The degree of mixing between bright and dark states depends on $B_{x}$ and $g_{e, h}$, whereby the intensity of $X_{b 1,2}$ $\left(X_{d 1,2}\right)$ is decreasing (increasing) with increasing field. By calculating the eigenvalues of Eq. (8) (see Sec. IV of the Supplemental Material [37]) we obtain four equations to fit our measurement data. For the fitting we use the measured values for $\delta_{0}=110 \mu \mathrm{eV}$ and $\delta_{1}=4.1 \mu \mathrm{eV}$. The value of $\delta_{2}$ for a dark exciton doublet is not known, as in all measured QDs only the $X_{d 1}$ component becomes visible, while $X_{d 2}$ stays dark (see the inset in Fig. 4). However, the dark-state splitting is supposed to be small, and we assume $\delta_{2} \approx 0$. Additionally, we find that within a range of $0-20 \mu \mathrm{eV}$ the influence of $\delta_{2}$ on the mixing is negligible. We obtain from the fit $\left|g_{e, x}\right|=0.27$ and $\left|g_{h, x}\right|=0.05$. Furthermore, we use the eigenvectors of Eq. (8) to derive the relative oscillator strength (ROS) $\mathcal{R}_{d 1,2}$ of $X_{d 1,2}$ (see Sec. V of the Supplemental Material [37]), which determines the coupling between dark and bright states. With the measured values for $\delta_{0}$ and $\delta_{1}$ given above, $\delta_{2}=0$, and the fitted values of $g_{e, x}=0.27$ and $g_{h, x}=-0.05$, we obtain the blue and red curves in Fig. 4. Note that we choose the sign of $g_{e, x}$ and $g_{h, x}$ so that the ROS becomes maximal for one component. As expected, the ROS

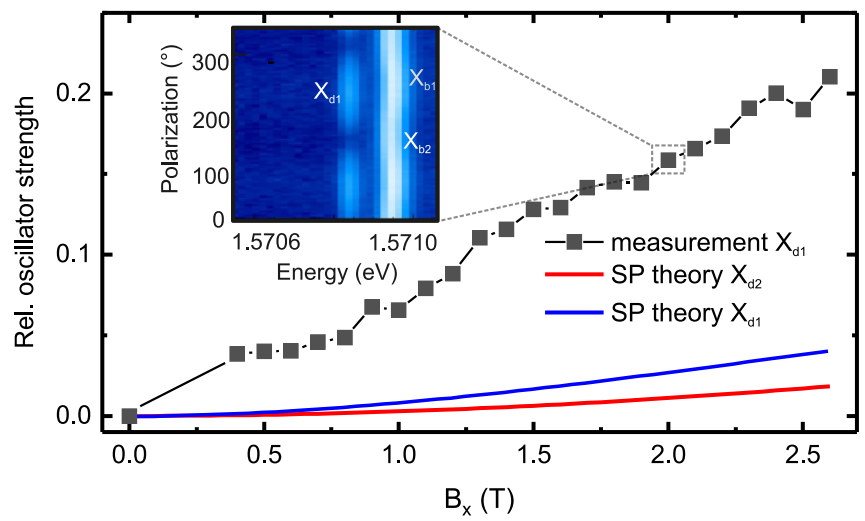

FIG. 4. Relative oscillator strength of the dark exciton state vs magnetic field along [110]. The black squares are the measured values for one dark exciton component $\left(X_{d 1}\right)$; the second component $\left(X_{d 2}\right)$ stays dark. The blue (red) curve is the calculated relative oscillator strength using the $e^{-}$and $h^{+}$in-plane $g$-factors extracted by fitting the measurement data via the SP Zeeman Hamiltonian. The inset shows linear-polarization-resolved spectra for $B_{x}=2 \mathrm{~T}$. 
(a)

(b)
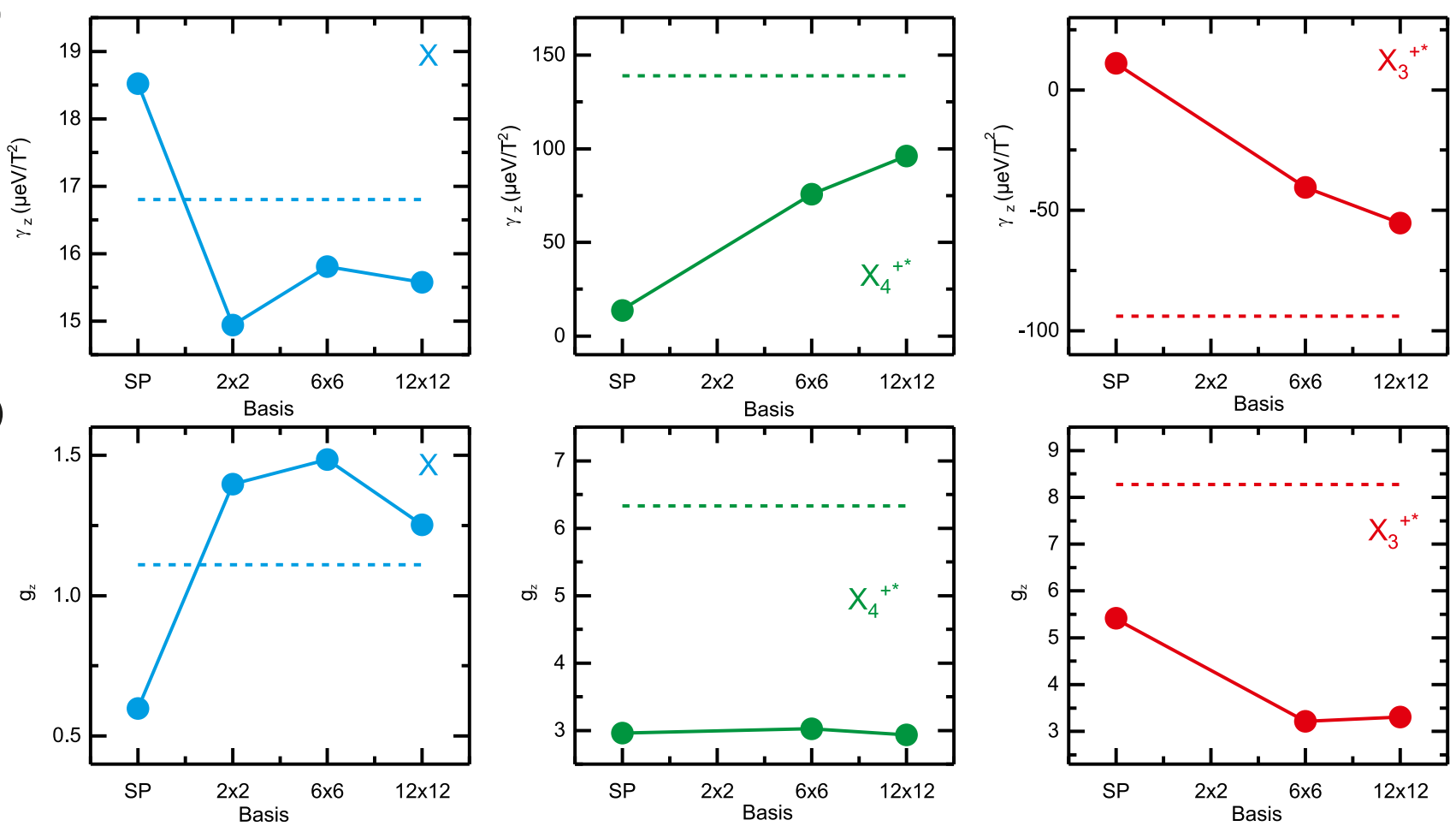

FIG. 5. Magnetic parameters of the ground-state exciton $(X)$ and the excited trion states $X_{4}^{+*}$ and $X_{3}^{+*}$ confined in a GaAs QD, calculated with the CI method using single-particle states obtained through the $\mathbf{k} \cdot \mathbf{p}$ method for a magnetic field along [001] (see the circles). The results for $\gamma_{z}$ are given in panel (a) and for $g_{z}$ in (b) for single-particle transitions (marked by SP on the horizontal axis) and for a two-electron and two-hole (marked by $2 \times 2$ ), a six-electron and six-hole $(6 \times 6)$, and a twelve-electron and twelve-hole $(12 \times 12)$ CI basis. Note that the effect of correlation increases with the basis size, i.e., from SP to a $(12 \times 12)$ CI basis. The dashed lines show the experimental results given in Table I.

of $X_{d 1,2}$ is increasing by ramping up the magnetic field, due to increased mixing between dark and bright $X$ states.

We also calculate from the measurement data the ROS according to

$$
\mathcal{R}_{d 1}=\frac{I_{X_{d 1}}}{I_{X_{d 1}}+I_{X_{b 1}}}
$$

where $I_{X_{d 1, b 1}}$ is the intensity of $X_{d 1, b 1}$. The result is shown in Fig. 4 (black rectangles). Obviously, the measured data do not correspond to the calculated ROS, and the coupling between bright and dark states is stronger than expected.

If we use now the ROS equations obtained via the eigenvectors of Eq. (8) (see Sec. V of the Supplemental Material [37]) and fit the measured trend in Fig. 4, we obtain $g_{h, x} \approx$ $-g_{e, x} \approx 0.52$, which is different from the result obtained by fitting the energy shift with the eigenvalues. Hence, the SP model is not self-consistent, and we can conclude that it is not valid in the weak confinement regime. Interestingly, the SP model yields reasonable results for strongly confining GaAs/AlGaAs QDs [32].

\section{CONFIGURATION-INTERACTION CALCULATIONS}

To gain a deeper insight into the experimental results and support our claim that the SP model is not suitable to extract single-particle $g$-factors from PL measurements, we perform calculations combining the eight-band $\mathbf{k} \cdot \mathbf{p}$ method for the computation of single-particle states and the CI method for the excitonic states confined in our weakly confining QDs. On the one hand, this approach allows a realistic treatment of the QD shape and composition [50,51], including strain and piezoelectricity up to second order [52-54]. On the other hand, it allows an intrinsic treatment of correlation effects, which are included in CI [31,51] via the excited SP states used to construct the Slater determinants. This is important, because we expect correlation effects between the confined carriers to play a dominant role in the weak confinement regime. We note that the eight-band $\mathbf{k} \cdot \mathbf{p}$ intrinsically introduces in our description LH and spin-orbit-split-off states, and furthermore causes the mixing of the total angular momentum $J= \pm 1 / 2$ and $\pm 3 / 2$ bulk spin states.

The simulated QD is composed of pure GaAs embedded in an $\mathrm{Al}_{0.4} \mathrm{Ga}_{0.6} \mathrm{As}$ matrix. Its shape reflects the results of atomic force microscopy measurements on droplet etched nanoholes fabricated under the same growth conditions as the QDs. Additionally, we optimized the structure to match the $X$ emission energy. The final QD shape is such that the QD top is convex while its base is concave (see Sec. VI of the Supplemental Material [37]) [50].

We start out by calculating the SP recombination of $X$ under a magnetic field along $z$, neglecting Coulomb interaction and correlation. Using Eq. (6) we can extract $\gamma, g_{2, z}$, and $g_{z}$ from the computed eigenvalues. The results are presented in Fig. 5. The value of $g_{z}=0.6$ [Fig. 5(b), left panel] as well as $\gamma=19 \mu \mathrm{eV} / \mathrm{T}^{2}$ [Fig. 5(a), left panel] show a significant 
difference from the measurement data. In the next step, we include CI in the simulation, where we start with a SP basis including two $e^{-}$and two $h^{+}(2 \times 2)$ states and increase it up to twelve $e^{-}$and twelve $h^{+}(12 \times 12)$ states. In the CI calculation, $\gamma\left(g_{z}\right)$ decreases (increases) by almost onequarter (a factor of 2) and approaches a value of $15 \mu \mathrm{eV} / \mathrm{T}^{2}$ (1.25), which is in good agreement with the measurement. We attribute the decrease of the diamagnetic coefficient to the fact that correlation effects lead to a "shrinkage" of the $X$ wave function compared to the bare single-particle states, so as to improve the overlap of $e^{-}$and $h^{+}$wave functions. Furthermore, our calculations point out that the SP model fails to describe the magneto-optical properties in the weak confinement regime, and correlation effects cannot be neglected even for the $X$ ground state.

In addition, we use CI to calculate the $g$-factors and diamagnetic coefficients for the states provided in Table I. The results are summarized in the same table (for details on the calculation, see Sec. VI of the Supplemental Material [37]), whereby we extend our analysis also to magnetic fields along $x$. The $X^{+} g$-factor shows only a small $x / z$ anisotropy, which is in good agreement with the experiment. Furthermore, the $g$-factors of the $X^{-}$state are $\approx 2$ times larger than that of the $X^{+}$state, which is not in agreement with the SP description as already discussed in Sec. II.

For the hot trions, the CI calculation provides an insight into the origin of large values of $\gamma_{z}$ and anomalous diamagnetic shift (see Fig. 5, middle and right panels). These phenomena stem from the mixing of the singlet $\left(X_{4}^{+*}\right)$ and the triplet $\left(X_{3}^{+*}\right)$ trion excited state, an effect that can be traced back to the different magnitude of the electron-hole exchange interaction experienced by each of the two holes constituting hot trion states [55]. The described effect can happen, e.g., if the excited trion state is spread over a larger region compared to the final hole state. This is the case of the weak confinement that occurs for our QDs in their lateral dimension, hence the anomalous diamagnetic shift is seen in our case for the Faraday configuration of the applied $B$ field. On the other hand, our QDs are much thinner in the vertical direction, and both holes in hot trions are then more strongly confined, with the result that they experience the exchange interaction with an electron with equal magnitude. Hence, no large diamagnetic shift is expected for Voigt configuration, exactly as we observe. We stress that the described mixing of a singlet and triplet of $X^{+}$for the $z$-direction is a purely multiparticle effect (not describable on an SP level), and moreover it occurs because of the small energy separation between $X_{4}^{+*}$ and $X_{3}^{+*}$ as seen in our experiments and calculations, where that amounts to $\approx 100 \mu \mathrm{eV}$ (see Sec. VI of the Supplemental Material [37]) [31,55]. On the other hand, the value of $g_{z}$ is mostly determined by the Zeeman splitting of the final SP hole states that are subtracted from trions (see also Sec. VI of the Supplemental Material [37]).

In general, the simulation results are qualitatively in good agreement with the measurement data. We partly attribute the mismatch in the absolute values between measurement and calculation to differences in the shape and size of the measured and simulated QD. Nevertheless, the CI also shows some deviations from the experiment: Due to the strong confinement along $z$, we would expect similar values for $\gamma_{x}$ for all states, which is in line with the measurement. However, we obtain for $X_{3}^{+*}$ a $\gamma_{x}=2 \mu \mathrm{eV} / \mathrm{T}^{2}$ from the calculations, which is about three times smaller than expected. Here the $\gamma_{x}$ value directly obtained from the SP states obtained by $\mathbf{k} \cdot \mathbf{p}$ is closer to the experimental one (see Fig. 10 of the Supplemental Material [37]). Also the calculated values of $g_{x}$ of $X$ and $X^{+}$are substantially larger than the measured ones. Finally, we note that the calculated binding energy of $X^{+}$with respect to the $X$ is only $600 \mu \mathrm{eV}$, whereby all measured QDs show a value of $\approx 2.5 \mathrm{meV}$. The reason for this deviation is not fully understood yet, but since the problem was observed in independent CI calculations [56], we speculate that it may stem from an intrinsic limitation of the CI method.

\section{CONCLUSION}

In conclusion, we have presented a comprehensive analysis of the optical transitions in a GaAs QD under above-bandgap excitation. The performed measurements allow us to determine the charge complexes forming the excited states in a QD. Furthermore, we provide an analysis of the $g$-factors and diamagnetic coefficients of the excited complexes in our GaAs/AlGaAs QDs obtained by droplet etching. On this basis, we are able to experimentally prove that the SP Zeeman Hamiltonian [21] cannot be used to reliably extract singleparticle $g$-factors from measurements of optical transitions in weakly confining QDs. CI calculations clearly show that interactions between the confined carriers-such as correlation effects-significantly influence the magneto-optical properties. The model calculations are able to quantitatively reproduce most of the observed values of diamagnetic shifts and $g$-factors not only for the neutral excitons, but also for some of the charged complexes observed experimentally. Some significant differences between experiment and calculation results are still present, which deserve further consideration in the future.

\section{ACKNOWLEDGMENTS}

We thank L. Vukusic, G. Katsaros, F. Binder, and B. Swolo for fruitful discussions and technical assistance. This work has been supported by the Austrian Science Fund (FWF): P29603, the Linz Institute of Technology (LIT), and the LIT Secure and Correct Systems Lab, financed by the state of Upper Austria. P.K and D.C received national funding from project CEITEC 2020 (LQ1601) with financial support from the Ministry of Education, Youth and Sports of the Czech Republic under the National Sustainability Programme II. Project CUSPIDOR has received funding from the QuantERA ERA-NET Cofund in Quantum Technologies implemented within the European Union's Horizon 2020 Programme. In addition, this project has received national funding from the MEYS and funding from European Union's Horizon 2020 (2014-2020) research and innovation framework programme under Grant Agreement No. 731473. P.K. was (partially) funded by project EMPIR 17FUN06 Siqust. This project has received funding from the EMPIR programme cofinanced by the Participating States and from the European Union's Horizon 2020 research and innovation programme. 
[1] Z. M. Wang, B. L. Liang, K. A. Sablon, and G. J. Salamo, Appl. Phys. Lett. 90, 113120 (2007).

[2] C. Heyn, A. Stemmann, T. Köppen, C. Strelow, T. Kipp, M. Grave, S. Mendach, and W. Hansen, Appl. Phys. Lett. 94, 18 (2009).

[3] Y. H. Huo, A. Rastelli, and O. G. Schmidt, Appl. Phys. Lett. 102, 152105 (2013).

[4] L. Schweickert, K. D. Jöns, K. D. Zeuner, S. F. Covre da Silva, H. Huang, T. Lettner, M. Reindl, J. Zichi, R. Trotta, A. Rastelli, and V. Zwiller, Appl. Phys. Lett. 112, 093106 (2018).

[5] D. Huber, M. Reindl, Y. Huo, H. Huang, J. S. Wildmann, O. G. Schmidt, A. Rastelli, and R. Trotta, Nat. Commun. 8, 15506 (2017).

[6] M. Reindl, J. H. Weber, D. Huber, C. Schimpf, S. F. Covre da Silva, S. L. Portalupi, R. Trotta, P. Michler, and A. Rastelli, Phys. Rev. B 100, 155420 (2019).

[7] E. Schöll, L. Hanschke, L. Schweickert, K. D. Zeuner, M. Reindl, S. F. Covre da Silva, T. Lettner, R. Trotta, J. J. Finley, K. Müller, A. Rastelli, V. Zwiller, and K. D. Jöns, Nano Lett. 19, 2404 (2019).

[8] J. Liu, R. Su, Y. Wei, B. Yao, S. Filipe, Y. Yu, J. Iles-smith, K. Srinivasan, A. Rastelli, J. Li, and X. Wang, Nat. Nanotechnol. 14, 586 (2019).

[9] R. Keil, M. Zopf, Y. Chen, B. Höfer, J. Zhang, F. Ding, and O. G. Schmidt, Nat. Commun. 8, 15501 (2017).

[10] D. Huber, M. Reindl, S. F. Covre da Silva, C. Schimpf, J. Martín-Sánchez, H. Huang, G. Piredda, J. Edlinger, A. Rastelli, and R. Trotta, Phys. Rev. Lett. 121, 033902 (2018).

[11] M. Gurioli, Z. Wang, A. Rastelli, T. Kuroda, and S. Sanguinetti, Nat. Mater. 18, 799 (2019).

[12] S. Stobbe, T. W. Schlereth, S. Höfling, A. Forchel, J. M. Hvam, and P. Lodahl, Phys. Rev. B 82, 233302 (2010).

[13] D. Huber, M. Reindl, J. Aberl, A. Rastelli, and R. Trotta, J. Opt. 20, 073002 (2018).

[14] E. Peter, P. Senellart, D. Martrou, A. Lemaître, J. Hours, J. M. Gérard, and J. Bloch, Phys. Rev. Lett. 95, 067401 (2005).

[15] D. Loss and D. P. DiVincenzo, Phys. Rev. A 57, 120 (1998).

[16] I. Schwartz, E. R. Schmidgall, L. Gantz, D. Cogan, E. Bordo, Y. Don, M. Zielinski, and D. Gershoni, Phys. Rev. X 5, 011009 (2015).

[17] L. Gaudreau, A. Bogan, M. Korkusinski, S. Studenikin, D. G. Austing, and A. S. Sachrajda, Semicond. Sci. Technol. 32, 093001 (2017).

[18] H. M. G. A. Tholen, J. S. Wildmann, A. Rastelli, R. Trotta, C. E. Pryor, E. Zallo, O. G. Schmidt, P. M. Koenraad, and A. Y. Silov, Phys. Rev. B 99, 195305 (2019).

[19] A. J. Bennett, M. A. Pooley, Y. Cao, N. Sklöd, I. Farrer, D. A. Ritchie, and A. J. Shields, Nat. Commun. 4, 1522 (2013).

[20] G. Medeiros-Ribeiro, E. Ribeiro, and H. Westfahl, Jr., Appl. Phys. A 77, 725 (2003).

[21] M. Bayer, G. Ortner, O. Stern, A. Kuther, A. A. Gorbunov, A. Forchel, P. Hawrylak, S. Fafard, K. Hinzer, T. L. Reinecke, S. N. Walck, J. P. Reithmaier, F. Klopf, and F. Schäfer, Phys. Rev. B 65, 195315 (2002).

[22] H. M. G. A. Tholen, J. S. Wildmann, A. Rastelli, R. Trotta, C. E. Pryor, E. Zallo, O. G. Schmidt, P. M. Koenraad, and A. Y. Silov, Phys. Rev. B 94, 245301 (2016).

[23] W. Sheng, Appl. Phys. Lett. 96, 133102 (2010).
[24] B. J. Witek, R. W. Heeres, U. Perinetti, E. P. A. M. Bakkers, L. P. Kouwenhoven, and V. Zwiller, Phys. Rev. B 84, 195305 (2011).

[25] Y. H. Huo, B. J. Witek, S. Kumar, J. R. Cardenas, J. X. Zhang, N. Akopian, R. Singh, E. Zallo, R. Grifone, D. Kriegner, R. Trotta, F. Ding, J. Stangl, V. Zwiller, G. Bester, A. Rastelli, and O. G. Schmidt, Nat. Phys. 10, 46 (2014).

[26] A. Ulhaq, Q. Duan, E. Zallo, F. Ding, O. G. Schmidt, A. I. Tartakovskii, M. S. Skolnick, and E. A. Chekhovich, Phys. Rev. B 93, 165306 (2016).

[27] M. C. Löbl, L. Zhai, J.-P. Jahn, J. Ritzmann, Y. Huo, A. D. Wieck, O. G. Schmidt, A. Ludwig, A. Rastelli, and R. J. Warburton, Phys. Rev. B 100, 155402 (2019).

[28] T. Takagahara, Phys. Rev. B 47, 4569 (1993).

[29] J. Shumway, A. Franceschetti, and A. Zunger, Phys. Rev. B 63, 155316 (2001).

[30] A. Schliwa, M. Winkelnkemper, and D. Bimberg, Phys. Rev. B 79, 075443 (2009).

[31] P. Klenovský, P. Steindl, and D. Geffroy, Sci. Rep. 7, 45568 (2017).

[32] S. Kunz, Magneto-optical properties of individual GaAs/AlGaAs quantum dots grown by droplet epitaxy, Ph.D. thesis, Institut National des Sciences Appliquées de Toulouse, 2013, http://www.theses.fr/2013ISAT0003.

[33] N. I. Cade, H. Gotoh, H. Kamada, H. Nakano, and H. Okamoto, Phys. Rev. B 73, 115322 (2006).

[34] K. V. Kavokin, Phys. Status Solidi A 195, 592 (2003).

[35] Y. Igarashi, M. Shirane, Y. Ota, M. Nomura, N. Kumagai, S. Ohkouchi, A. Kirihara, S. Ishida, S. Iwamoto, S. Yorozu, and Y. Arakawa, Phys. Rev. B 81, 245304 (2010).

[36] T. Warming, E. Siebert, A. Schliwa, E. Stock, R. Zimmermann, and D. Bimberg, Phys. Rev. B 79, 125316 (2009).

[37] See Supplemental Material at http://link.aps.org/supplemental/ 10.1103/PhysRevB.100.235425 for further details.

[38] M. Bayer, S. N. Walck, T. L. Reinecke, and A. Forchel, Phys. Rev. B 57, 6584 (1998).

[39] C. Schulhauser, D. Haft, R. J. Warburton, K. Karrai, A. O. Govorov, A. V. Kalameitsev, A. Chaplik, W. Schoenfeld, J. M. Garcia, and P. M. Petroff, Phys. Rev. B 66, 193303 (2002).

[40] M.-F. Tsai, H. Lin, C.-H. Lin, S.-D. Lin, S.-Y. Wang, M.-C. Lo, S.-J. Cheng, M.-C. Lee, and W.-H. Chang, Phys. Rev. Lett. 101, 267402 (2008).

[41] Y. J. Fu, S. D. Lin, M. F. Tsai, H. Lin, C. H. Lin, H. Y. Chou, S. J. Cheng, and W. H. Chang, Phys. Rev. B 81, 113307 (2010).

[42] J. van Bree, A. Y. Silov, P. M. Koenraad, M. E. Flatté, and C. E. Pryor, Phys. Rev. B 85, 165323 (2012).

[43] J. van Bree, A. Y. Silov, M. L. van Maasakkers, C. E. Pryor, M. E. Flatté, and P. M. Koenraad, Phys. Rev. B 93, 035311 (2016).

[44] A. Schwan, B.-M. Meiners, A. Greilich, D. R. Yakovlev, M. Bayer, A. D. B. Maia, A. A. Quivy, and A. B. Henriques, Appl. Phys. Lett. 99, 221914 (2011).

[45] C. E. Pryor and M. E. Flatté, Phys. Rev. Lett. 96, 026804 (2006).

[46] H. Watzinger, C. Kloeffel, L. Vukusic, M. D. Rossell, V. Sessi, J. Kukucka, R. Kirchschlager, E. Lausecker, A. Truhlar, M. Glaser, A. Rastelli, A. Fuhrer, D. Loss, and G. Katsaros, Nano Lett. 16, 6879 (2016).

[47] I. A. Akimov, K. V. Kavokin, A. Hundt, and F. Henneberger, Phys. Rev. B 71, 075326 (2005). 
[48] M. Durnev, M. Glazov, and E. Ivchenko, Physica E 44, 797 (2012).

[49] E. A. Chekhovich, A. B. Krysa, M. S. Skolnick, and A. I. Tartakovskii, Phys. Rev. Lett. 106, 027402 (2011).

[50] S. Birner, T. Zibold, T. Andlauer, T. Kubis, M. Sabathil, A. Trellakis, and P. Vogl, IEEE Trans. Electron Dev. 54, 2137 (2007).

[51] P. Klenovský, A. Schliwa, and D. Bimberg, Phys. Rev. B 100, 115424 (2019).

[52] A. Beya-Wakata, P. Y. Prodhomme, and G. Bester, Phys. Rev. B 84, 195207 (2011).
[53] P. Klenovský, P. Steindl, J. Aberl, E. Zallo, R. Trotta, A. Rastelli, and T. Fromherz, Phys. Rev. B 97, 245314 (2018).

[54] J. Aberl, P Klenovský, J. S. Wildmann, J. Martín-Sánchez, T. Fromherz, E. Zallo, J. Humlíček, A. Rastelli, and R. Trotta, Phys. Rev. B 96, 045414 (2017).

[55] M. Z. Maialle and M. H. Degani, Phys. Rev. B 76, 115302 (2007).

[56] L. Wang, V. Křápek, F. Ding, F. Horton, A. Schliwa, D. Bimberg, A. Rastelli, and O. G. Schmidt, Phys. Rev. B 80, 085309 (2009). 